\title{
Impact of connective tissue disease on the surgical outcomes of aortic dissection in patients with cystic medial necrosis
}

\author{
Toshiki Fujiyoshi ${ }^{4}$, Kenji Minatoya ${ }^{1}$, Yoshihiko Ikeda $^{2}$, Hatsue Ishibashi-Ueda ${ }^{2}$, Takayuki Morisaki ${ }^{3}$, \\ Hiroko Morisaki ${ }^{3}$ and Hitoshi Ogino ${ }^{4}$
}

\begin{abstract}
Background: A retrospective analysis was performed to determine the impact of genetically diagnosed connective tissue disease (CTD) on the early and late outcomes of surgical treatment for aortic dissection in patients having aortic pathology associated with cystic medial necrosis (CMN).

Methods: Between 2003 and 2013, a total of 43 patients (37 \pm 12.8 years old, 23 men, 20 women) who had undergone surgery for aortic dissection associated with $\mathrm{CMN}$ in the aortic wall underwent genetic examinations. Subsequently, there were 30 patients with CTD (CTD group) and 13 without CTD (non-CTD group).

Results: There were no early or late deaths (the follow-up rate was 100\% for $57.1 \pm 43.0$ months). The median age was significantly lower in the CTD group $(p=0.030)$. The rate of elastic fiber loss was significantly higher in the CTD group $(p=0.014)$. In the long-term follow-up, there were no significant differences in the incidences of re-dissection $(p=0.332)$. However, re-operations were required more frequently in the CTD group ( $p=0.037)$.

Conclusions: In patients with CTD as well as CMN, the onset of aortic dissection tends to be earlier, which would result in higher rates of re-operation, compared with the non-CTD group. Closer and stricter follow-up with medication and adequate surgical treatments with appropriate timing are mandatory for such high-risk patients.
\end{abstract}

Keywords: Aortic dissection, Surgery, Genetically diagnosed connective tissue disease, Cystic medial necrosis

\section{Background}

Cystic medial necrosis (CMN) or degeneration is found in surgical specimens of aortic dissection, and tends to be associated with higher risks of various aortic complications including aortic dissection and dilatation [1-3]. In addition, CMN is also considered as one of the histological markers for connective tissue diseases (CTD), including Marfan syndrome, Loeys-Dietz syndrome, and Ehlers-Danlos syndrome. Although some differences are found in the degree of loss of elastic fibers, there are no significant differences in the histopathological patterns within the aortic wall between CTD and non-CTD patients, especially in patients who are of advanced age and have systemic hypertension for a long time $[4,5]$.

\footnotetext{
* Correspondence: fff.toshiki@gmail.com

${ }^{4}$ Cardiovascular Surgery, Tokyo Medical University, 6-7-1 Nishishinjuku

Shinjuku-ku Tokyo, 160, Tokyo -0023, Japan

Full list of author information is available at the end of the article
}

Further genetic examinations are then highly recommended to diagnose CTD more precisely. However, for every patient with aortic dissection, there are difficulties in performing routine genetic examinations which are generally carried out when association with CTD is suspected, based on bodily features and family histories. On the other hand, there have been few published studies looking at the adverse effects and relationships of CTD and $\mathrm{CMN}$ to the surgical outcomes of aortic dissection. Under these circumstances, we wanted to determine the impact of genetically diagnosed CTD on the early and late outcomes of surgical treatment for aortic dissection in patients having pathologies associated with CMN.

\section{Methods}

\section{Patient profiles}

We reviewed our institutional database to identify patients who underwent initial surgery for aortic dissection 
between April 2003 and March 2013 at the National Cerebral and Cardiovascular Center, Osaka, Japan. A total of 321 patients underwent initial surgery for aortic dissection and 298 patients $(64.0 \pm 15.7$ years old, $56 \%$ male) underwent postoperative pathological examinations of the surgical specimen during this period, in which $\mathrm{CMN}$ was present in 141 patients (47.3\%). Of them, 43 patients $(37.0 \pm 12.8$ years old, $53 \%$ male) subsequently underwent genetic examinations. Our criteria for genetic examination were as follows: (1) patients with bodily features consistent with the current Ghent criteria, (2) young patients under 50 years of age, and (3) patients with family history of aortic diseases. This study was approved by the National Cerebral and Cardiovascular Center of Japan Institutional Review Board waived the need for individual patient consent under the provisions for deidentified human subject and quality improvement research.

The patient characteristics are shown in Table 1. Thirty patients (69.8\%, CTD group) were diagnosed genetically as having CTD, whereas the remaining 13 patients (30.2\%, non-CTD group) did not have any genetic disorders. The details of genetic disorders are shown in Table 2. Preoperative variables were compared between the CTD and non-CTD groups (Table 3). The median age at the onset of aortic dissection was significantly lower in the CTD group, namely 33.5 years in the CTD group vs. 42.0 years in the non-CTD $(p=0.030)$. In addition, there were differences in the Stanford classification of aortic dissection $(p=0.015)$ and the stages of aortic dissection $(p=0.009)$ between the two patient groups. In the CTD group, there were type A aortic dissections in 11 patients (37\%, 7 acute and 4 chronic) and type B aortic dissections in 19 patients (63\%, 1 acute and 18 chronic). In the non-CTD group, there were type A aortic dissections in 10 patients $(77 \%, 9$ acute and 1 chronic) and type B in 3 patients (23\%, 3 chronic). The incidence of type $\mathrm{B}$ aortic dissection was higher in the CTD group. Two patients in the CTD group developed

Table 1 Patient characteristics

\begin{tabular}{ll}
\hline Patient characteristics $(n=43)$ & $23: 20$ \\
\hline Male: Female & $37.0 \pm 12.8$ \\
Mean age at AD (years) & \\
Stanford AD classification & $21(48.8 \%)$ \\
$\quad$ Type A & $22(51.2 \%)$ \\
$\quad$ Type B & \\
Stage of aortic dissection & $17(39.5 \%)$ \\
Acute & $26(60.5 \%)$ \\
Chronic & \\
Connective tissue disorder ${ }^{\mathrm{a}} 30(69.8 \%)$ & \\
\hline $\begin{array}{l}\text { a } D \text { aortic dissection } \\
\text { genetically diagnosed }\end{array}$
\end{tabular}

Table 2 Genetic disorders in CTD

\begin{tabular}{ll}
\hline Genetic disorders in CTD & Number \\
\hline FBN1 & 19 \\
TGFBR2 & 4 \\
ACTA2 & 3 \\
MYH11 & 1 \\
SMAD3 & 1 \\
TGFB2 & 1 \\
COLIAI & 1 \\
\hline
\end{tabular}

CTD genetically diagnosed connective tissue disease

three-channel aortic dissection, although it was not found in the non-CTD group. The incidence of family history of aortic dissection was significantly higher in the CTD group ( $57 \%$ vs. $23 \%, p=0.043$ ). The rate of preoperative renal dysfunction (serum creatinine $\geq 2.0$ ) was significantly higher in the non-CTD group $(p=0.028)$.

In these series, the surgical treatments were performed according to the same indication criteria at the single center with the same surgical team staff. In the acute phase, emergent or urgent surgeries were carried out, in cases with acute type A aortic dissection with patent false channels excluding intramural hematoma, and with complicated acute type B aortic dissection. In patients with chronic aortic dissection or with residual aortic dissection in the long term after the initial surgical replacement, surgical interventions were indicated when the

Table 3 Preoperative variables

\begin{tabular}{|c|c|c|c|}
\hline Preoperative variables $(n=43)$ & $\mathrm{CTD}(n=30)$ & $\begin{array}{l}\text { non-CTD } \\
(n=13)\end{array}$ & $p$ value \\
\hline Male & $14(46.7 \%)$ & $9(69.2 \%)$ & $\underline{0.303}$ \\
\hline $\begin{array}{l}\text { Median age at AD (years } \\
\text { [range]) }\end{array}$ & $33.5[19-65]$ & $42.0[28-71]$ & 0.030 \\
\hline \multicolumn{4}{|l|}{ Stanford AD classification } \\
\hline Type A & $11(36.7 \%)$ & $10(76.9 \%)$ & 0.015 \\
\hline Type B & $19(63.3 \%)$ & $3(23.1 \%)$ & \\
\hline \multicolumn{4}{|l|}{ Aortic pathology } \\
\hline Acute & $8(26.7 \%)$ & $9(69.2 \%)$ & 0.009 \\
\hline Chronic & $22(73.3 \%)$ & $4(30.8 \%)$ & \\
\hline Three-channel AD & $2(6.7 \%)$ & none & $\underline{0.518}$ \\
\hline Family history of $A D$ & $17(56.7 \%)$ & $3(23.1 \%)$ & 0.043 \\
\hline \multicolumn{4}{|l|}{$\begin{array}{l}\text { Other coexisting } \\
\text { conditions }\end{array}$} \\
\hline Hypertension & $16(53.3 \%)$ & $8(61.5 \%)$ & $\underline{0.870}$ \\
\hline Hyperlipidemia & $1(3.3 \%)$ & $2(15.4 \%)$ & $\underline{0.440}$ \\
\hline Diabetes mellitus & none & $1(7.7 \%)$ & $\underline{0.677}$ \\
\hline CKD $(\mathrm{Cr} \geq 2.0)$ & none & $2(15.4 \%)$ & 0.028 \\
\hline Smoker & $9(30.0 \%)$ & $5(38.5 \%)$ & 0.850 \\
\hline
\end{tabular}

chronic kidney disease, $\mathrm{Cr}$ creatinine value, NS no significant difference 
aortic maximum diameter exceeded 50 to $55 \mathrm{~mm}$. Root repairs such as aortic valve-sparing surgery or composite valve-graft root replacement were carried out with the site of the root over 40 to $45 \mathrm{~mm}$, predominantly for the CTD patients [6]. In 2 patients described above, surgical treatments were indicated for 3-channel dissection, even though the diameter was less than $50 \mathrm{~mm}$.

\section{Histopathological examination}

The samples were surgically resected from the dissecting aortic wall. Histopathological examination consisted of light microscopy on the surgical specimen stained with hematoxylin-eosin stain, Masson's trichrome stain, elastica van Gieson stain, and toluidine blue stain. CMN was diagnosed if the aorta displayed fragmentation of elastic fibers associated with cystic accumulation of the extracellular matrix. The degree of loss of elastic fibers was defined as elastin fragmentation in the media stained with elastica van Gieson using a diagram of the grading system. Three grades were recognized as follows: grade 0 or $1+$ (no loss or minimal loss or mild fragmentation of elastic fibers), grade 2+ (intermediate change), and grade 3+ (complete loss of elastic fibers in some fullthickness portions of the media) (Fig. 1) [5]. Afterwards,

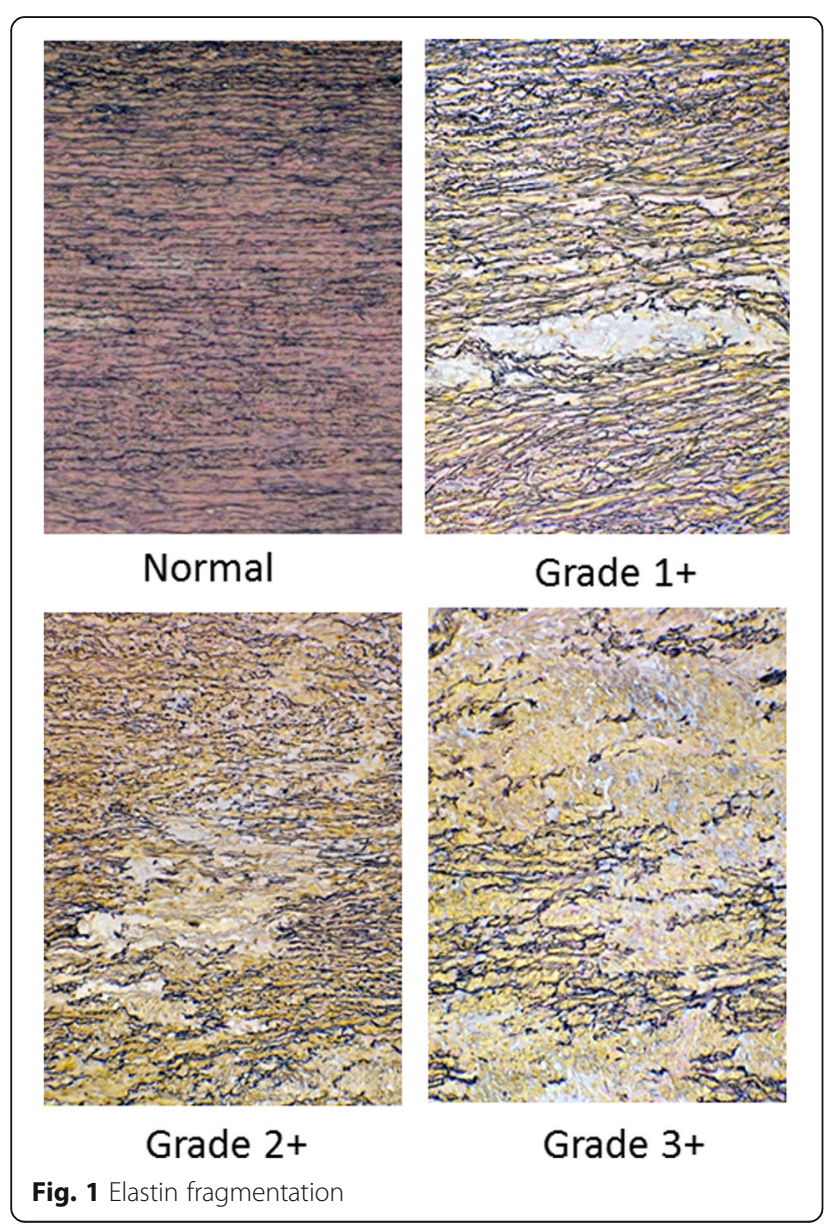

loss of elastic fibers was combined into two clinically relevant categories, namely, (1) no or minimal loss of elastic fibers (grade 0 or $1+$ representing no loss or minimal loss or mild fragmentation of elastic fibers), and (2) loss of elastic fibers (grade $2+$ or $3+$ representing a significant loss of elastic fibers in some portion of the media) $[5,7]$.

\section{Genetic examination (sequencing and mutation analysis)} Mutation analyses were performed by bidirectional Sanger sequencing of exons and exon-intron boundaries of FBN1, TGFBR1, and TGFBR2 genes first [8-11]. PCR products were purified and sequenced using BigDye Terminator chemistry v.1.1 on an ABI Prism3130xl or 3730xl (Applied Biosystems). In cases in which mutations of FBN1 gene were not found, mutations were further examined with the multiple ligation probe amplification method on an ABI Prism3130xl (Applied Biosystems). In cases in which these two methods failed to find mutations in patients, SMAD3, ACTA2, and TGFB2 genes were additionally analyzed by bidirectional Sanger sequencing of exons and exon-intron boundaries. For patients without mutations in FBN1, TGFBR1, TGFBR2, SMAD3, ACTA2, or TGFB2 genes, exome sequencing was performed after TruSeq Exome enrichment on HiSeq1000 (Illumina) for searching mutations in MYH11, COL3A1, COLIAI (COL1A1), and COL1A2 genes [12-16]. Nonsense, missense, or splicing variations in these genes were further analyzed by Sanger sequencing if they were not present in SNP databases, predicted to be damaging by PolyPhen-2, or the SIFT program, or previously reported to be a pathogenic mutation.

\section{Follow-up}

Data were collected from hospital admission and outpatient medical records. All patients were regularly followed, either at our center or at other local hospitals. All of them underwent strict blood pressure control before leaving the hospital, with $\beta$-blockers in all cases and angiotensin II receptor blockers in some cases. The follow-up rate was $100 \%$ and the duration was $57.1 \pm$ 43.0 (range, 6-132) months.

\section{Statistical analysis}

Two-tailed Student's $t$ tests for continuous variables and chi-square tests for categorical variables were used to make univariate comparisons between the two groups. The long-term outcomes including re-dissection and re-operation were analyzed using Kaplan-Meier methods and compared with log-rank tests. The risk factors (see Appendix) for mortality and late aortic re-operations were estimated using a multivariate proportional hazard regression analysis (Cox model). A $p$ value of $<0.05$ was considered statistically significant. SPSS software, version 
22.0 for Windows (IBM SPSS Inc., Chicago, IL) was used for all calculations.

In this study, between the CTD and the non-CTD groups, the early and late outcomes of the initial aortic surgery for aortic dissection were compared to demonstrate the impact of CTD on the surgical outcomes in the patients with aortic dissection associated with CMN.

\section{Results}

\section{Initial surgeries for aortic dissection}

As shown in Table 4, in the CTD group, there were initial surgical repairs of the ascending aorta in 5 patients and the aortic arch in 9 patients. Simultaneous aortic root repairs were performed in 11 of these 14 patients. The others underwent repairs of the descending aorta (in 10 patients), the thoracoabdominal aorta (in 2 patients), and the abdominal aorta (in 4 patients). In the non-CTD group, the surgical repairs were for the ascending aorta in 3 patients and the aortic arch in 7 patients. Simultaneous aortic root repair was carried out in 1 patient. The other 3 patients underwent repairs of the descending aorta (in 1 patient) and the thoracoabdominal aorta (in 2 patients). There were concomitant mitral valve surgeries in 2 patients in the CTD group.

\section{Histopathological findings}

CMN classification showed minimal or no loss of elastic fibers in 3 patients $(10 \%)$ and loss of elastic fibers in 27 patients $(90 \%)$ of 30 CTD patients, whereas minimal or no loss of elastic fibers was found in 6 patients $(46.2 \%)$ and loss of elastic fibers in 7 patients (53.8\%) of 13 non-

\begin{tabular}{|c|c|c|c|}
\hline Initial surgery $(n=43)$ & CTD $(n=30)$ & $\begin{array}{l}\text { non-CTD } \\
(n=13)\end{array}$ & $p$ value \\
\hline \multicolumn{4}{|l|}{ Surgical site of aorta } \\
\hline Ascending aorta & $5(16.7 \%)$ & $3(23.1 \%)$ & 0.944 \\
\hline Aortic arch & $9(30.0 \%)$ & $7(53.8 \%)$ & $\underline{0.253}$ \\
\hline Descending aorta & $10(33.3 \%)$ & $1(7.7 \%)$ & $\underline{0.164}$ \\
\hline $\begin{array}{l}\text { Thoraco-abdominal } \\
\text { aorta }\end{array}$ & $2(6.7 \%)$ & $2(15.4 \%)$ & $\underline{0.740}$ \\
\hline Abdominal aorta & $4(13.3 \%)$ & none & $\underline{0.417}$ \\
\hline $\begin{array}{l}\text { Simultaneous aortic } \\
\text { root }\end{array}$ & $11(36.7 \%)$ & $1(7.7 \%)$ & $\underline{0.115}$ \\
\hline $\begin{array}{l}\text { Concomitant mitral } \\
\text { valve surgery }\end{array}$ & $2(6.7 \%)$ & none & $\underline{0.869}$ \\
\hline \multicolumn{4}{|c|}{ Conditions of false channel after the initial surgery } \\
\hline Double barrel & $23(76.7 \%)$ & $8(61.5 \%)$ & $\underline{0.519}$ \\
\hline Partial thrombosed & $2(6.7 \%)$ & $2(15.4 \%)$ & $\underline{0.740}$ \\
\hline Thrombosed (IMH) & $5(16.7 \%)$ & $3(23.1 \%)$ & 0.945 \\
\hline
\end{tabular}

CTD genetically diagnosed connective tissue disease, NS no significant difference, $I M H$ intramural hematoma
CTD patients. The rate of loss of elastic fibers was significantly higher in the CTD group $(p=0.014)$.

\section{Postoperative data}

There were no early deaths. In the long term, despite of there being no late deaths, new aortic dissection occurred at the same sites (three-channel aortic dissection) in 3 patients or at different sites in 3 patients (a total of 6 patients [20.0\%] of the CTD group), whereas only 1 patient $(7.7 \%)$ in the non-CTD group developed it at a different site $(p=0.310)$. The absence of re-dissection was not significantly different between the two groups (log-rank, $p=0.380$ ) (Fig. 2), that is, $83.2 \%$ in the CTD group and $85.7 \%$ in the non-CTD at 5 years.

In contrast, in all, there were no significant differences in the incidences of re-dissection and re-operation, when all of the patients were divided into the two patient groups according to the grade of CMN. Of 43 patients, 9 patients had no or minimal loss of elastic fibers (Grade 1+) and 34 patients had loss of elastic fibers (Grade $2+$ or $3+$ ). There were no occurrences of re-dissection and 5 (55.6\%) patients required re-operation in the former group, whereas 7 patients (20.6\%) developed re-dissection and 25 patients (73.5\%) required re-operation in the latter group. However, interestingly, all 7 patients who suffered from aortic re-dissection had high-grade $\mathrm{CMN}$, that is, loss of elastic fibers (Grade $2+$ or $3+$ ); these 7 patients consisted of $6(22.2 \%)$ of 27 CTD patients and $1(14.7 \%)$ of 7 non-CTD patients $(p=0.951)$.

There were no significant differences in the re-operation rates between the Stanford aortic dissection classification groups $(p=0.332)$, that is, 13 of 21 with type A $(62.0 \%)$ and 17 of 22 with type B (77.3\%). There were also no significant differences in the absence of aortic re-operation (log-rank, $p=0.404$ ), that is, $13.8 \%$ with type $\mathrm{A}$ and $14.5 \%$ with type $B$ at 5 years. In contrast, re-operations were required more frequently $(p=0.037)$; there were 24

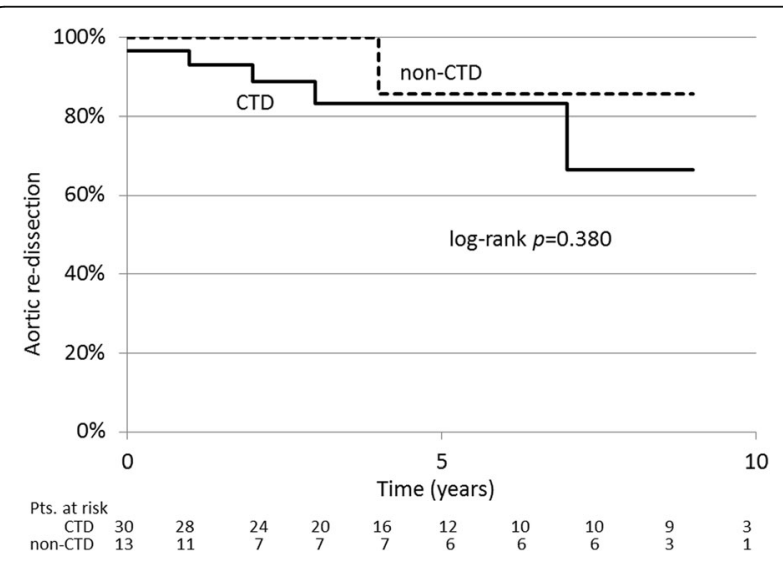

Fig. 2 Absence of aortic re-dissection (Kaplan-Meier). (CTD = genetically diagnosed connective tissue disease) 
(80.0\%) patients in the CTD group versus 6 patients (46.0\%) in the non-CTD group. The absence of aortic reoperation tended to be worse (log-rank, $p=0.052$ ), that is, $7.9 \%$ in the CTD group and $31.0 \%$ in the non-CTD group at 5 years (Fig. 3). Twelve of 24 CTD patients (50.0\%) and 3 of 6 non-CTD patients (50.0\%) required thirdly surgical interventions. Despite no substantial differences in the initial and following surgical procedures between the two patient groups, the rate of surgical interventions to the descending aorta was significantly higher in the CTD group $(p=0.021)$, that is, 16 patients (53.3\%) versus 2 patients (15.4\%). The rate of surgical interventions to the aortic root was also significantly higher in the CTD group $(p=0.037)$, that is, 15 patients $(50.0 \%)$ versus 2 patients $(15.4 \%)$. Regarding the time frame for aortic re-interventions, 15 of 24 patients $(62.5 \%)$ in the CTD group underwent re-operations during the two years of follow-up after the initial surgery, compared with 3 of 6 patients $(50.0 \%)$ in the non-CTD group. Univariate analysis revealed that patent false channel $(p=0.019)$, and simultaneous aortic root repair $(p=0.024)$ were significant risk factors for re-operation. In the multivariate analysis of risk factors for re-operation, patent false channel, simultaneous aortic root repair, and preoperative systemic hypertension were independent risk factors (Table 5).

\section{Discussion}

CMN is often observed in the resected aorta in patients with CTD and tends to be associated with the worst long-term prognoses; this also causes aortic dilatation and dissection in non-CTD patients as well as in CTD patients $[1,17,18]$. However, it is difficult to demonstrate pathologically remarkable differences in the fragmentation of elastic fibers and accumulation of extracellular matrix in the formation of the cystic structures between CTD and non-CTD patients, especially in elderly patients with systemic hypertension $[4,5]$. In this study, we hypothesized

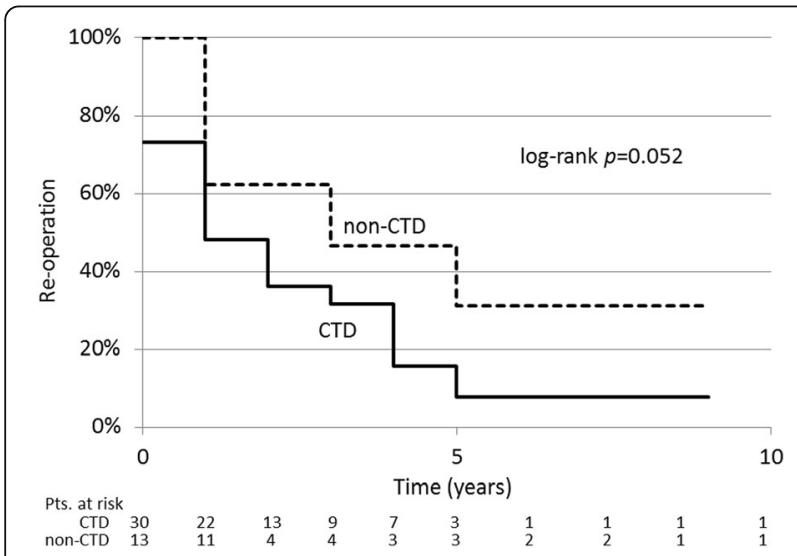

Fig. 3 Absence of aortic re-operation (Kaplan-Meier). (CTD = genetically diagnosed connective tissue disease)
Table 5 Multivariate analysis of risk foctors for re-operation

\begin{tabular}{lccc}
\hline Variables & HR & $95 \% \mathrm{Cl}$ & $p$ value \\
\hline Patient-related risk factors & & & \\
$\quad$ Hypertension & 3.45 & $1.12-10.63$ & 0.031 \\
$\begin{array}{l}\text { Dissection-related risk factor } \\
\quad \text { Patent false channel }\end{array}$ & 5.22 & $1.37-19.89$ & 0.016 \\
$\begin{array}{l}\text { Procedure-related risk factor } \\
\quad \text { Simultaneous aortic root repair }\end{array}$ & 4.31 & $1.37-13.60$ & 0.013 \\
\hline
\end{tabular}

$H R$ hazard ratio, $\mathrm{Cl}$ confidence i

that coexistence of genetically identified CTD would be associated with worse postoperative prognoses after surgeries for aortic dissection in patients with CMN. To elucidate it, we compared early and late outcomes, including occurrence of new aortic events after the initial surgeries for aortic dissection of CMN-positive patients, between the CTD and non-CTD groups.

CMN might be due to long-term hemodynamic forces (hypertension) and age $[4,5]$. It was demonstrated that patients with CMN have increased risks of serious vascular complications [19, 20]. However, few studies have seemed to look at adverse effects of CMN on the surgical outcomes of aortic dissection, as far as we investigated. With regard to findings of histological examinations, Trotter and Olsen reported that the degrees of elastic fragmentation were variable in Marfan patients, and can also be found even in non-Marfan subjects [4]. Nakajima et al. pointed out a higher degree of elastic fiber loss or fragmentation in Marfan patients, compared with non-Marfan patients having CMN [21]. Similarly, in our study, the grade of loss or fragmentation of elastic fibers was higher in the CTD patients than that of the non-CTD patients. Clinically, in this study, the age of the onset of initial aortic dissection was also significantly younger in the CTD patients, and aortic re-dissection occurred only in the patients with high-grade CMN, that is, loss of elastic fibers. Such clinical phenomena may be affected directly by such fragility of the aortic walls due to higher degree of loss or fragmentation of elastic fibers in the aortic wall. Consequently, the presence of coexisting CTD should be suspected or examined genetically in relatively young patients with higher grades of loss or fragmentation of elastic fibers in the aortic wall.

With regard to occurrence of re-dissection in the long term after the initial aortic dissection, the incidence was higher in CTD patients, despite no significant statistical differences. Interestingly, in the CTD group, 2 patients had suffered from three-channel dissection before the initial surgery and another 3 patients developed it in the long term after surgery; this may show a more severe fragility of the degenerative aortic wall in CTD patients. In the histopathological examinations, the grades of loss or fragmentation of elastic fibers were higher in CTD 
patients than in those of the non-CTD group. Looking at the relationship between the histopathological findings and the re-dissection rate, there were no significant differences between patients with no or minimal loss of elastic fibers and those with loss of elastic fibers. However, interestingly, all of the CTD and non-CTD patients suffering from aortic re-dissection had high-grade CMN, that is, loss of elastic fibers in the histopathological examinations. Consequently, it is important to estimate the long-term outcome after initial surgery for aortic dissection according to the results of genetic examinations as well as the histopathological findings.

In contrast, regarding re-operations, in CTD patients, higher rates of redo surgery were reported than in nonCTD patients [22, 23]. In our study, redo surgeries were also required more frequently in CTD patients. In particular, the rate of descending aortic repairs was significantly higher, compared with non-CTD patients. In patients with $\mathrm{CMN}$ combined with CTD, higher onset rates of type $\mathrm{B}$ aortic dissection were demonstrated than the others [24]. Our study also demonstrated the higher rate of type B aortic dissection in CTD patients. Related to this issue, Schoenhoff et al. reported that $86 \%$ of Marfan patients with type $B$ aortic dissection required redo surgery for residual aortic enlargement [25]. In our study, similar findings were revealed. In the CTD group, $80.0 \%$ of the patients required re-operations after the initial surgical repairs in the long term, $72.7 \%$ in type A and $84.2 \%$ in type B.

The rate of surgical interventions to the aortic root was also significantly higher in the CTD group. Progressive enlargement of the aortic root is one of the characteristics of CTD patients, which is related directly to the early onset of type A aortic dissection. In cases with aortic root enlargement of 40 to $50 \mathrm{~mm}$ in diameter, prophylactic root repairs such as aortic valve-sparing surgery or composite valve-graft root replacement are recommended in the guidelines to eliminate risks of ruptured type A aortic dissection [26]. Progressive aortic root enlargement also should be one of the reasons for redo surgery after various surgical repairs for type A or B aortic dissection, whether it is associated with aortic dissection or not. In our study, a total of $39.5 \%$ of all patients underwent aortic root repairs; this included $63.6 \%$ of CTD patients and $20.0 \%$ of nonCTD patients with type A aortic dissection, and $42.1 \%$ of CTD patients with type B aortic dissection. Moreover, simultaneous aortic root repair was a significant risk factor for re-operation in the univariate and multivariate analyses. Consequently, these circumstances resulted in the higher rates of redo surgeries in the CTD patients.

Obviously, there are some limitations to this study. First, this is a retrospective study dealing with a small number of enrolled patients. In particular, the number of the patients undergoing genetic examinations was too small to elucidate exactly the impact of CTD on the outcomes of surgeries for aortic dissection. Aortic dissection was also variable, such as acute or chronic, and type A or B. Apart from the histological aortic pathologies, there are other factors or requirements for redo surgeries after initial surgeries for aortic dissection, relating to types of aortic dissection, conditions of the false channels, surgical techniques, extent of repairs, and so on. The surgical procedures of the initial operations for aortic dissection were variable, which depended on a variety of conditions, including the stages of aortic dissection, such as acute and chronic, and the settings of the surgery, such as emergent/urgent and elective. Theoretically, the re-operation rates become significantly higher in cases with no tear resection and patent false channel $[27,28]$. Actually, there may be some differences in the incidences of re-operation between limited ascending/hemiarch replacement and entire arch replacement. The rates should also be different after the total arch replacement between with or without elephant trunk procedures on the distal anastomosis site [29]. The conditions of aortic dissection, including the patency of the false channel, were also variable. More detailed studies dealing with a larger number of patients is required to elucidate precisely the impact of genetically identified CTD on the surgical outcomes of aortic dissection in patients with CMN.

Finally, most $(90.0 \%)$ of the CTD patients showed high-grade CMN, that is, loss of elastic fibers. However, similar findings were also found in a half $(53.8 \%)$ of nonCTD patients. The genetic examinations are still limited by the issue of availability. There might be other different CTDs with unknown genetic characteristics. Consequently, in case of high-grade CMN, it is necessary to recognize, even without genetically diagnosed CTD at present, potentially high-risk subjects who would develop re-dissection and require re-operations at relatively shorter intervals after surgery for aortic dissection.

\section{Conclusions}

Patients with CTD as well as CMN in the aortic wall tend to develop aortic dissection, particularly of type B, at an earlier age. They require re-operation more frequently due to enlargement of the residual dissecting aorta and/or the aortic root, compared with non-CTD patients. Therefore, closer and stricter follow-up with medication and with earlier and more adequate surgical treatments including more extended repairs should be considered for such high-risk patients having CTD and $\mathrm{CMN}$, and for some non-CTD patients having highgrade $\mathrm{CMN}$.

Abbreviations

CMN: Cystic medial necrosis; CTD: Connective tissue disease 


\section{Acknowledgements}

Not applicable.

\section{Funding}

None.

\section{Availability of data and materials}

Please contact author for data requests.

\section{Authors' contributions}

TF carried out the molecular genetic studies, participated in the sequence alignment and drafted the manuscript. TM and HM carried out the genetic examinations. YI and HIU carried out the histopathological examination. TF participated in the design of the study and performed the statistical analysis. $\mathrm{HO}$ and $\mathrm{KM}$ conceived of the study, and participated in its design and coordination and helped to draft the manuscript. All authors read and approved the final manuscript.

\section{Ethics approval and consent to participate}

This study was approved by the National Cerebral and Cardiovascular Center of Japan Institutional Review Board waived the need for individual patient consent under the provisions for identified human subject and quality improvement research.

\section{Consent for publication}

The patients consented to participate and publication of this case report.

\section{Competing interests}

The authors declare that they have no competing interests.

\section{Publisher's Note}

Springer Nature remains neutral with regard to jurisdictional claims in published maps and institutional affiliations.

\section{Author details}

${ }^{1}$ Departments of Cardiovascular Surgery, National Cerebral and Cardiovascular Center, Osaka, Japan. ${ }^{2}$ Departments of Pathology, National Cerebral and Cardiovascular Center, Osaka, Japan. ${ }^{3}$ Departments of Bioscience and Genetics, National Cerebral and Cardiovascular Center, Osaka, Japan. ${ }^{4}$ Cardiovascular Surgery, Tokyo Medical University, 6-7-1 Nishishinjuku Shinjuku-ku Tokyo, 160, Tokyo -0023, Japan.

Received: 15 June 2017 Accepted: 10 November 2017 Published online: 23 November 2017

\section{References}

1. Girdauskas E, Kuntze T, Borger MA, Doenst T, Mochalski M, Walther T, et al. Long-term prognosis of type a aortic dissection in non-Marfan patients with histologic pattern of cystic medial necrosis. Ann Thorac Surg. 2008;85(3):972-7.

2. Marsalese DL, Moodie DS, Lytle BW, Cosgrove DM, Ratliff NB, Goormastic M, et al. Cystic medial necrosis of the aorta in patients without Marfan's syndrome: surgical outcome and long-term follow-up. J Am Coll Cardiol. 1990;16(1):68-73.

3. Larson EW, Edwards WD. Risk factors for aortic dissection: a necropsy study of 161 cases. Am J Cardiol. 1984;53(6):849-55.

4. Trotter SE, Olsen EG. Marfan's disease and Erdheim's cystic medionecrosis. A study of their pathology. Eur Heart J. 1991;12(1):83-7.

5. Schlatmann TJ, Becker AE. Histologic changes in the normal aging aorta: implications for dissecting aortic aneurysm. Am J Cardiol. 1977;39(1):13-20.

6. Tanaka H, Ogino H, Matsuda H, Minatoya K, Sasaki H, Iba Y. Midterm outcome of valve-sparing aortic root replacement in inherited connective tissue disorders. Ann Thorac Surg. 2011;92(5):1646-9.

7. Roberts WC, Vowels TJ, Ko JM, Filardo G, Hebeler RF Jr, Henry AC, et al. Comparison of the structure of the aortic valve and ascending aorta in adults having aortic valve replacement for aortic stenosis versus for pure aortic regurgitation and resection of the ascending aorta for aneurysm. Circulation. 2011;123(8):896-903.

8. Morisaki H, Akutsu K, Ogino H, Kondo N, Yamanaka I, Tsutsumi Y, et al. Mutation of ACTA2 gene as an important cause of familial and nonfamilial nonsyndromatic thoracic aortic aneurysm and/or dissection (TAAD). Hum Mutat. 2009:30(10):1406-11.

9. Dietz HC, Cutting GR, Pyeritz RE, Maslen CL, Sakai LY, Corson GM, et al. Marfan syndrome caused by a recurrent de novo missense mutation in the fibrillin gene. Nature. 1991;352(6333):337-9.

10. Mizuguchi T, Collod-Beroud G, Akiyama T, Abifadel M, Harada N, Morisaki T, et al. Heterozygous TGFBR2 mutations in Marfan syndrome. Nat Genet. 2004;36(8):855-60

11. Loeys BL, Chen J, Neptune ER, Judge DP, Podowski M, Holm T, et al. A syndrome of altered cardiovascular, craniofacial, neurocognitive and skeletal development caused by mutations in TGFBR1 or TGFBR2. Nat Genet. 2005: 37(3):275-81.

12. De Paepe A, Devereux RB, Dietz HC, Hennekam RC, Pyeritz RE. Revised diagnostic criteria for the Marfan syndrome. Am J Med Genet. 1996; 62(4):417-26.

13. Guo DC, Papke CL, Tran-Fadulu V, Regalado ES, Avidan N, Johnson RJ, et al. Mutations in smooth muscle alpha-actin (ACTA2) cause coronary artery disease, stroke, and Moyamoya disease, along with thoracic aortic disease. Am J Hum Genet. 2009;84(5):617-27.

14. Superti-Furga A, Gugler E, Gitzelmann R, Steinmann B. Ehlers-Danlos syndrome type IV: a multi-exon deletion in one of the two COL3A1 alleles affecting structure, stability, and processing of type III procollagen. J Biol Chem. 1988;263(13):6226-32

15. Zhu L, Vranckx R, Khau Van Kien P, Lalande A, Boisset N, et al. Mutations in myosin heavy chain 11 cause a syndrome associating thoracic aortic aneurysm/aortic dissection and patent ductus arteriosus. Nat Genet. 2006: 38(3):343-9.

16. Pannu H, Tran-Fadulu V, Papke CL, Scherer S, Liu Y, Presley C, et al. MYH11 mutations result in a distinct vascular pathology driven by insulin-like growth factor 1 and angiotensin II. Hum Mol Genet. 2007;16(20):2453-62.

17. Bähr M, Postler E, Meyermann R. Fatal stroke in a patient with carotid and middle cerebral artery dissection associated with cystic medial necrosis. J Neurol. 1996:243(10):722-3.

18. Takeda K, Matsumiya G, Nishimura M, Matsue H, Tomita Y, Sawa Y. Giant circumflex coronary artery aneurysm associated with cystic medial necrosis in a non-Marfan patient. Ann Thorac Surg. 2007;83(2):668-70.

19. Pande AK, Gosselin G, Leclerc Y, Leung TK. Aortic dissection complicating coronary angioplasty in cystic medial necrosis. Am Heart J. 1996;131:1221-3.

20. Isner JM, Donaldson RF, Fulton D, Bhan I, Payne DD, Cleveland RJ. Cystic medial necrosis in coarctation of the aorta: a potential factor contributing to adverse consequences observed after percutaneous balloon angioplasty of coarctation sites. Circulation. 1987;75:689-95.

21. Nakashima Y, Kurozumi T, Sueishi K, Tanaka K. Dissecting aneurysm: a clinicopathologic and histopathologic study of 111 autopsied cases. Hum Pathol. 1990;21(3):291-6.

22. Finkbohner R, Johnston D, Crawford ES, Coselli J, Milewicz DM. Marfan syndrome long-term survival and complications after aortic aneurysm repair. Circulation. 1995:91:728-33.

23. Iba Y, Minatoya K, Matsuda H, Sasaki H, Tanaka H, Ogino H, et al. Surgical experience with aggressive aortic pathologic process in Loeys-Dietz syndrome. Ann Thorac Surg. 2012;94(5):1413-7.

24. Cameron DE, Alejo DE, Patel ND, Nwakanma LU, Weiss ES, Vricella LA, et al. Aortic root replacement in 372 Marfan patients: evolution of operative repair over 30 years. Ann Thorac Surg. 2009;87:1344-9.

25. Scharfschwerdt M, Sievers HH, Greggersen J, Hanke T, Misfeld M. Prosthetic replacement of the ascending aorta increases wall tension in the residual aorta. Ann Thorac Surg. 2007:83(3):954-7.

26. Judge DP, Dietz HC. Marfan's syndrome. Lancet. 2005;366(9501):1965-76.

27. Heinemann $M$, Laas J, Borst HG, et al. Surgery extended into the aortic arch in acute type a dissection. Indications, techniques, and results. Circulation. 1991:84(5 Suppl):III25-30.

28. Girdauskas E, Kuntze T, Borger MA, Falk V, Mohr FW. Distal aortic reinterventions after root surgery in Marfan patients. Ann Thorac Surg. 2008; 86(6):1815-9.

29. Watanuki $\mathrm{H}$, Ogino $H$, Minatoya $K$, Matsuda $H$, Sasaki $H$, Ando M, et al. Is emergency total arch replacement with a modified elephant trunk techniquejustified for acute type a aortic dissection? Ann Thorac Surg. 2007; 84(5):1585-91. 